\title{
A locking-free and optimally convergent discontinuous-Galerkin-based extended finite element method for cracked nearly incompressible solids
}

Shen, Yongxing, yongxing.shen@sjtu.edu.cn, Shanghai Jiao Tong University

\begin{abstract}
For nearly incompressible elasticity, volumetric locking is a well-known phenomenon with low-order (cubic or lower) finite element method methods, of which continuous extended finite element methods (XFEMs) are no exception. We will present an XFEM that is simultaneously lock-free and optimally convergent. Based on our earlier work of an optimally convergent discontinuous-Galerkin-based XFEM, the method herein consists in enriching a region surrounding the crack tip that contains a fixed ball, i.e., the enrichment zone does not shrink with the mesh parameter, and the enrichment space consists of modes I and II asymptotic solutions without the use of partition of unity. To achieve a locking-free method, the discontinuous Galerkin method is used between all neighboring elements, and specially designed lifting operators are adopted whose lifting space and testing space are no longer polynomials but instead contain the singular strain and stress components, respectively.
\end{abstract}

